\title{
Estudio del nivel de protección contra la corrosión de un hormigón polimérico ${ }^{(\bullet)}$
}

\author{
E. García ${ }^{(*)}$ y E. Valle ${ }^{(*)}$
}

\begin{abstract}
Resumen El objetivo de este estudio es determinar a nivel laboratorio el grado de protección contra la corrosión que puede ofrecer un hormigón polimérico de base poliéster desarrollado en el Instituto de Investigaciones Eléctricas (IIE), empleando las técnicas electroquímicas de impedancia faradaica y ruido electroquímico.
\end{abstract}

Palabras clave: Hormigón. Evaluación electroquímica. Corrosión.

\section{Study on the degree of corrosion protection by a polimeric concrete}

\begin{abstract}
The main purpose of this article is to determine, at laboratory level, the degree of protection against corrosion that a polimeric concrete, developed in the Instituto de Investigaciones Eléctricas (IIE), can offer, employing the electrochemical impedance and electrochemical noise techniques.
\end{abstract}

Keywords: Concrete. Electrochemical evaluation. Corrosion.

\section{INTRODUCCIÓN}

El problema que plantea la corrosión de la sección enterrada de las patas de las torres de trasmisión de energía eléctrica es de gran importancia, porque lleva asociado el fallo de las mismas ocasionando pérdidas significativas.

En la actualidad, se ha pretendido minimizar esta situación embebiendo las patas de las torres en hormigón, partiendo de la suposición de que el hormigón presenta un $\mathrm{pH}$ muy alcalino, lo que ocasionaría que el acero se pasivara enérgicamente y que la difusión del agua al interior del hormigón fuera mínima. Suposiciones no del todo correctas, dado que estas condiciones pueden cambiar drásticamente como se explicará más adelante, originando, en consecuencia, la presencia de un alto nivel de corrosión.

Lo que se persigue en este primer estudio a nivel de laboratorio es determinar el grado de protección contra la corrosión que puede ofrecer un hormigón

(•) Trabajo recibido el día 19 de mayo de 1997.

(*) Instituto de Investigaciones Eléctricas. Avda. Reforma 113, Colonia Palmira, 62490 Temixco, Morelos (México). polimérico, de base poliéster, desarrollado por el Instituto de Investigaciones Eléctricas (IIE) (1).

Para ello, se aplicaron técnicas electroquímicas, básicamente impedancia faradaica, medición de potencial $\mathrm{y}$, finalmente, ruido electroquímico, tanto en potencial como en corriente. Esto fue indispensable porque la aplicación de la técnica de pérdida de peso es imposible llevarla a cabo en este sistema. Además, las técnicas electroquímicas ofrecen la posibilidad de realizar un seguimiento continuo de la corrosión sobre la misma probeta.

El hormigón proporciona al acero tanto protección física como química. La física consiste en que es una barrera "poco permeable" (2) que retarda el acceso de agentes agresivos a la superficie del acero de refuerzo. La química se debe a la elevada alcalinidad que presenta este medio (2), provocando en la superficie del acero una capa pasiva protectora.

Cuando las condiciones de servicio ocasionan que el hormigón se altere, a través de él penetran sustancias agresivas que dan lugar a que se desencadene la corrosión de las armaduras con las siguientes consecuencias (3):

- El acero disminuye de sección o, incluso, se convierte completamente en óxido, disminuyendo así su capacidad mecánica. 
- Los productos de corrosión, debido a que son más voluminosos que sus elementos de partida, generan tensiones que pueden agrietar el hormigón.

- La adherencia acero-hormigón disminuye o desaparece, debido a la naturaleza expansiva de los productos de corrosión.

La causa más frecuente de la pérdida de pasividad se debe a la penetración de iones agresivos, principalmente el ion cloruro, hacia la intercara acero-hormigón (3 y 4). La penetración de los iones cloruro produce una disminución del $\mathrm{pH}$ de la solución que se encuentra en los poros del hormigón (5) debido a la hidrólisis del cloruro de hierro formado como producto de corrosión. Por encima de una cierta concentración límite de iones cloruro, la capa pasiva del acero se deteriora como resultado de las picaduras producidas por este ion, originando así una corrosión inicialmente local, pero que puede llegar a ser generalizada.

\section{PROCEDIMIENTO EXPERIMENTAL}

Para obtener resultados experimentales se construyeron probetas de hormigón en las que se embebieron en cada una tres varillas de acero al carbono 1018 , teniendo cada varilla una superficie de exposición en el hormigón de $65 \mathrm{~cm}^{2}$ (Fig. 1). Las varillas se lijaron hasta grado $600 \mathrm{y}$, posteriormente, se desengrasaron con acetona. La primera varilla de cada probeta se utilizó como electrodo de trabajo, la segunda como contraelectrodo y la tercera como electrodo de referencia; en otros casos, como fue la medida del potencial de corrosión de las varillas, se empleó como electrodo de referencia uno saturado de calomelanos (ESC).

Las probetas utilizadas en este trabajo se pueden clasificar en tres tipos diferentes:

- La construida con hormigón convencional.

- La construida con hormigón polimérico.

- La construida con hormigón convencional con un recubrimiento de un espesor de $7 \mathrm{~mm}$ de hormigón polimérico.

Cada uno de estos tipos se duplicó para determinar la reproducibilidad de los datos.

El medio en el que se sumergieron las probetas de hormigón fue una solución salina de $\mathrm{NaCl}$ al 3 $\%$ a temperatura ambiente.

Como se dijo anteriormente, se aplicaron dos técnicas electroquímicas que fueron la de impedancia faradaica y la de ruido electroquímico en potencial y corriente simultáneamente.

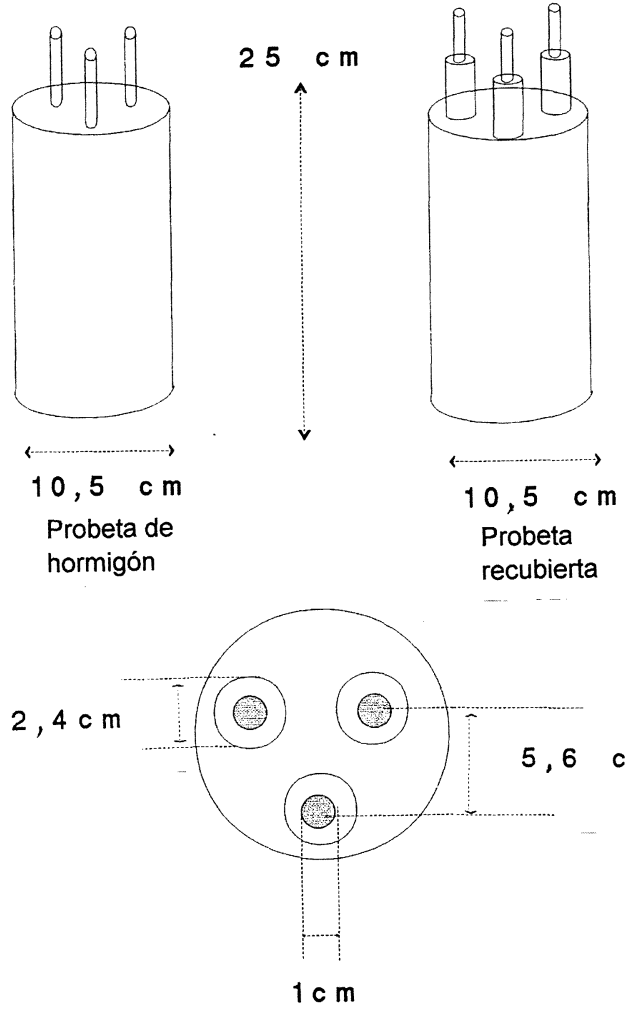

FIG. 1.-Esquema de las probetas.

FIG. 1.-Specimen scheme.

\subsection{Impedancia faradaica}

Los métodos que emplean corriente alterna para el estudio de los procesos de electrodo se conocen como métodos periódicos.

La corriente alterna puede pasar a través de la interfase metal-solución como consecuencia de la reacción electródica (corriente faradaica) o debido a la carga o descarga de la doble capa electroquímica (corriente no faradaica); la suma de ambas intensidades corresponde a la corriente total.

La estimulación llevada a cabo sobre las probetas de hormigón para la aplicación de la técnica de impedancia faradaica fue de $25 \mathrm{mV}$ de amplitud, barriendo un intervalo de frecuencias de 20.000 hasta $0,1 \mathrm{~Hz}$.

\subsection{Ruido electroquímico}

El ruido electroquímico es una técnica que consiste en registrar las fluctuaciones espontáneas de corriente y/o potencial que se presentan en un electrodo de trabajo. Existen dos tipos de ruido: uno en potencial y el otro en corriente. Ambos proporcionan información sobre la dinámica de los procesos que ocurren en la superficie del metal (6). 
Para la aplicación de esta técnica es necesario contar con un equipo capaz de adquirir datos de potencial y/o corriente, en períodos de tiempo que oscilen entre 0,25 y $1,5 \mathrm{~s}$ (6). Dichos datos se almacenan para su posterior análisis. A esta colección de datos obtenidos durante un intervalo de tiempo determinado se le denomina serie de tiempo.

En este trabajo se tomaron simultáneamente a circuito abierto, tanto los valores de la fluctuación de corriente como de potencial, con una velocidad de muestreo de $1 \mathrm{~s}$, para obtener series de tiempo con 2.060 datos y así poder determinar la resistencia de ruido $\left(R_{\mathrm{s}}\right)$, que es la inversa de la velocidad de corrosión.

La $R_{\mathrm{S}}(6)$ se puede definir como la relación existente entre la desviación estándar de la señal de potencial y la desviación estándar de la señal de corriente, según se expresa en la siguiente ecuación:

$$
R_{\mathrm{s}}=\frac{\sigma V}{\sigma I}
$$

Chen (7) llevó a cabo un análisis matemático fundamentado en los principios de la teoría electroquímica, encontrando que $R_{\mathrm{s}}$ es equivalente a la resistencia de polarización, $R_{\mathrm{p}}$, lográndose así algo que ya se había observado empíricamente para evaluar la velocidad de corrosión (8).

La aplicación del ruido electroquímico para obtener el valor de $R_{\mathrm{p}}$ cobró gran importancia a partir de los trabajos de Chen et al. (9), donde evaluaron con éxito el nivel de protección contra la corrosión de recubrimientos orgánicos, que presentan gran resistencia eléctrica, circunstancia muy similar para este sistema de hormigón polimérico, por lo cual se seleccionó como la técnica electroquímica más adecuada para determinar el grado de protección contra la corrosión que puede ofrecer este nuevo material.

\section{RESULTADOS EXPERIMENTALES Y DISCUSIÓN}

En la tabla I se presentan los valores de potencial de corrosión de las diferentes probetas frente a un ECS. Se aprecia claramente que las probetas de hormigón normal adquieren rápidamente un potencial inferior a $-500 \mathrm{mV}$, potencial que corresponde a un estado de corrosión, mientras que, tanto en las probetas de hormigón polimérico como en las recubiertas durante un período prolongado, no fue factible determinar un potencial de corrosión. Esto se puede deber a que el material polimérico aisla la varilla metálica del medio corrosivo, y sólo la probeta recubierta A presentó un tiempo inferior que hizo posible la medición de un potencial de corro-
TABLA I.- Valores del potencial de corrosión de las probetas, en $\mathrm{mV}$ (ESC)

TABLE I.-Corrosion potential of samples, $m V(S C E)$

\begin{tabular}{|c|c|c|c|c|c|c|}
\hline Días & \multicolumn{2}{|c|}{$\begin{array}{c}\text { Probetas } \\
\text { de hormigón }\end{array}$} & \multicolumn{2}{c|}{$\begin{array}{c}\text { Probetas } \\
\text { de hormigón } \\
\text { polimérico }\end{array}$} & \multicolumn{2}{c|}{$\begin{array}{c}\text { Probetas } \\
\text { recubiertas } \\
\text { de hormigón } \\
\text { polimérico }\end{array}$} \\
\hline & A & B & A & B & A & B \\
\hline 0 & -24 & -37 & - & - & - & - \\
1 & -487 & -448 & - & - & - & - \\
7 & -599 & -570 & - & - & - & - \\
14 & -580 & -574 & - & - & -557 & - \\
22 & -603 & -590 & 11 & 20 & -619 & 17 \\
41 & -596 & -599 & 0 & 0 & -100 & 0 \\
51 & -572 & -596 & -4 & -5 & -275 & -3 \\
59 & -565 & -596 & 0 & 0 & -236 & 0 \\
73 & -555 & -592 & -325 & -300 & -410 & -470 \\
99 & -650 & -712 & 45 & 104 & -473 & 100 \\
120 & -505 & -515 & -120 & -118 & -130 & -250 \\
\hline
\end{tabular}

sión, lo cual induce a pensar que el recubrimiento, posiblemente, presentaba defectos.

Tanto las probetas de hormigón polimérico como las recubiertas mostraron potenciales más nobles que las de hormigón normal de tal suerte que los valores de este potencial se desplazaron a niveles que corresponden a la pasivación, efecto debido seguramente a la naturaleza inhibitoria de la corrosión que ofrece el hormigón polimérico, circunstancia que se mostrará más adelante en las pruebas de impedancia faradaica.

La figura 2 presenta los diagramas de Nyquist del hormigón en solución de $\mathrm{NaCl}$ al $3 \%$ a diferentes tiempos, mostrando un semicírculo capacitivo y un elemento Warburg difusivo, mecanismo clásico para estos sistemas de acero-hormigón.

La figura 3 corresponde a las pruebas de impedancia faradaica a diferentes tiempos de las probetas de hormigón polimérico y de las recubiertas. En términos generales, se puede apreciar que la respuesta es muy similar durante todo el tiempo en los dos sistemas, presentando primeramente un semicírculo capacitivo correspondiente a la trasferencia de carga, relacionada con la reacción electroquímica de corrosión en la superficie de la varilla; posteriormente, se aprecia un semicírculo inductivo que indica una adsorción en la superficie de la varilla.

Circunstancia muy especial para estos sistemas aquí mencionados es la adsorción, que se puede deber a la presencia del polímero presente en el hormigón, el cual podría actuar adsorbiéndose en la superficie metálica intensamente produciendo el efecto de un inhibidor de corrosión.

Se puede apreciar que los valores de reactancia, tanto para el semicírculo capacitivo como inductivo, se incrementan significativamente al trascurrir 

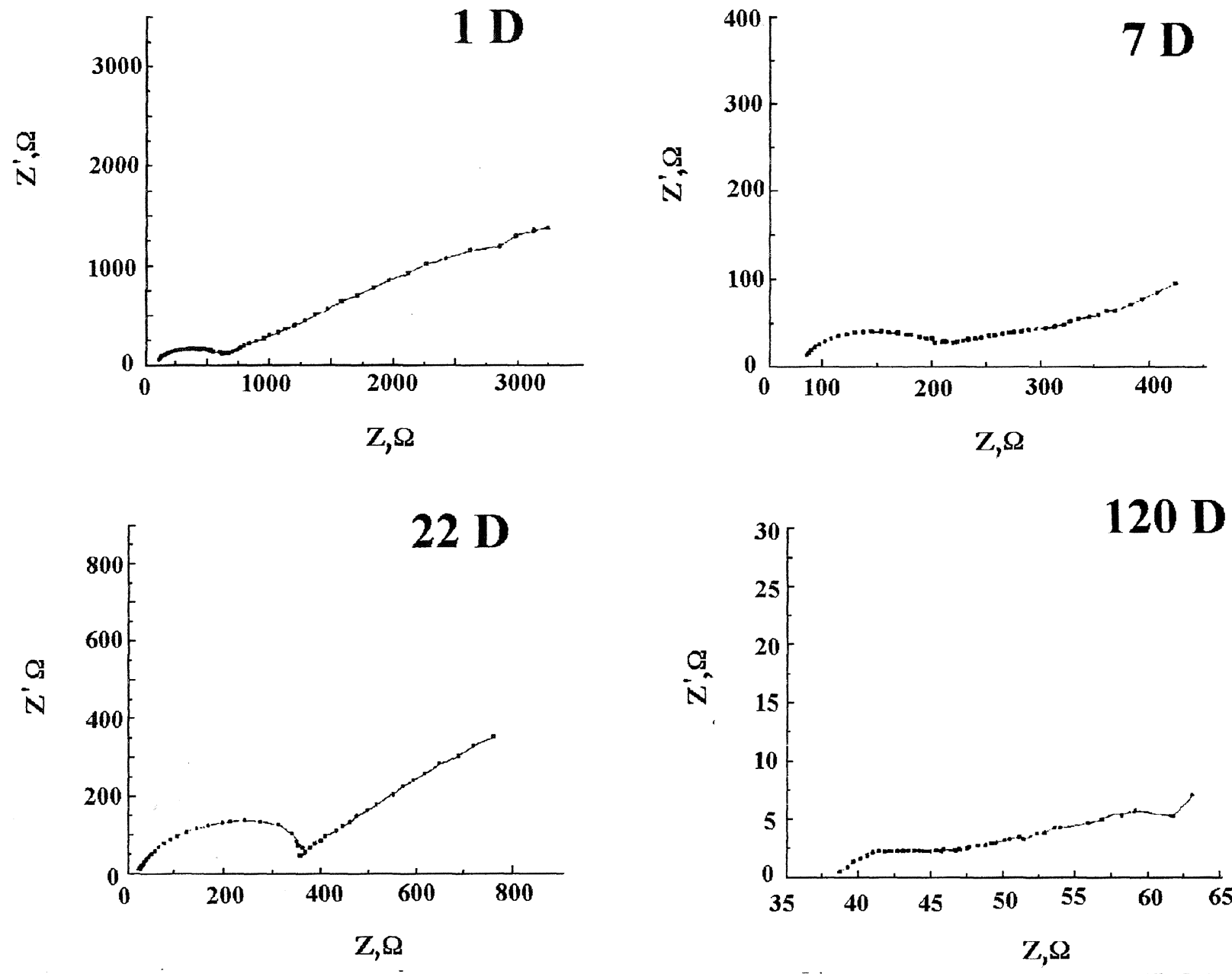

FIG. 2.-Ensayos de impedancia en probetas de hormigón normal a diferentes tiempos $\left(65 \mathrm{~cm}^{2}\right)$.

FIG. 2.-Impedance tests in normal concrete specimens at different times $\left(65 \mathrm{~cm}^{2}\right)$.

el tiempo, siendo alrededor de $5.000 \Omega$ a los 59 días, $12.000 \Omega$ a los 73 días y, finalmente, alrededor de $200.000 \Omega$ a los 120 días, indicando esto un mayor nivel de adsorción.

En la figura 4 se representa gráficamente la desviación estándar a diferentes tiempos de la señal de ruido en potencial de las probetas, mostrando claramente dos secciones. Una correspondiente a las probetas de hormigón y la otra sección a las probetas con protección de hormigón polimérico. En esta sección se nota un cambio importante en los valores, disminuyendo significativamente a los 59 días, coincidiendo esto con el momento en el que fue posible la aplicación de la técnica de impedancia faradaica en las probetas de hormigón polimérico y en las probetas recubiertas de hormigón polimérico, pudiendo considerar estos 59 días como el tiempo necesario para la permeación del electrólito.

La figura 5 corresponde a la desviación estándar en corriente de las probetas en estudio. Como en el caso anterior, se aprecia una gran reproducibilidad de los datos así como las dos secciones, correspondiendo una a las probetas de hormigón y la otra a las protegidas con hormigón polimérico, pero en esta ocasión se aprecia que la probeta recubierta $A$ (CPA) muestra un comportamiento que se separa en cierto grado de las restantes probetas con protección de hormigón polimérico, coincidiendo esto con la información que ofrece la tabla I que indica una mala aplicación del recubrimiento.

Finalmente, la figura 6 corresponde a los valores de $R_{\mathrm{p}}$ obtenidos de las señales de ruido en corriente y en potencial, donde se aprecia claramente el nivel de protección que confirió el hormigón polimérico a las varillas de acero, que es, al menos, de tres órdenes de magnitud, con excepción de la probeta recubierta $\mathrm{A}$ (CPA) con defectos de recubrimiento, donde el nivel de protección es menor.

\section{CONCLUSIONES}

- Se pudo determinar que el fenómeno de corrosión en hormigón es, básicamente, un fenómeno de difusión y de trasferencia de carga. 

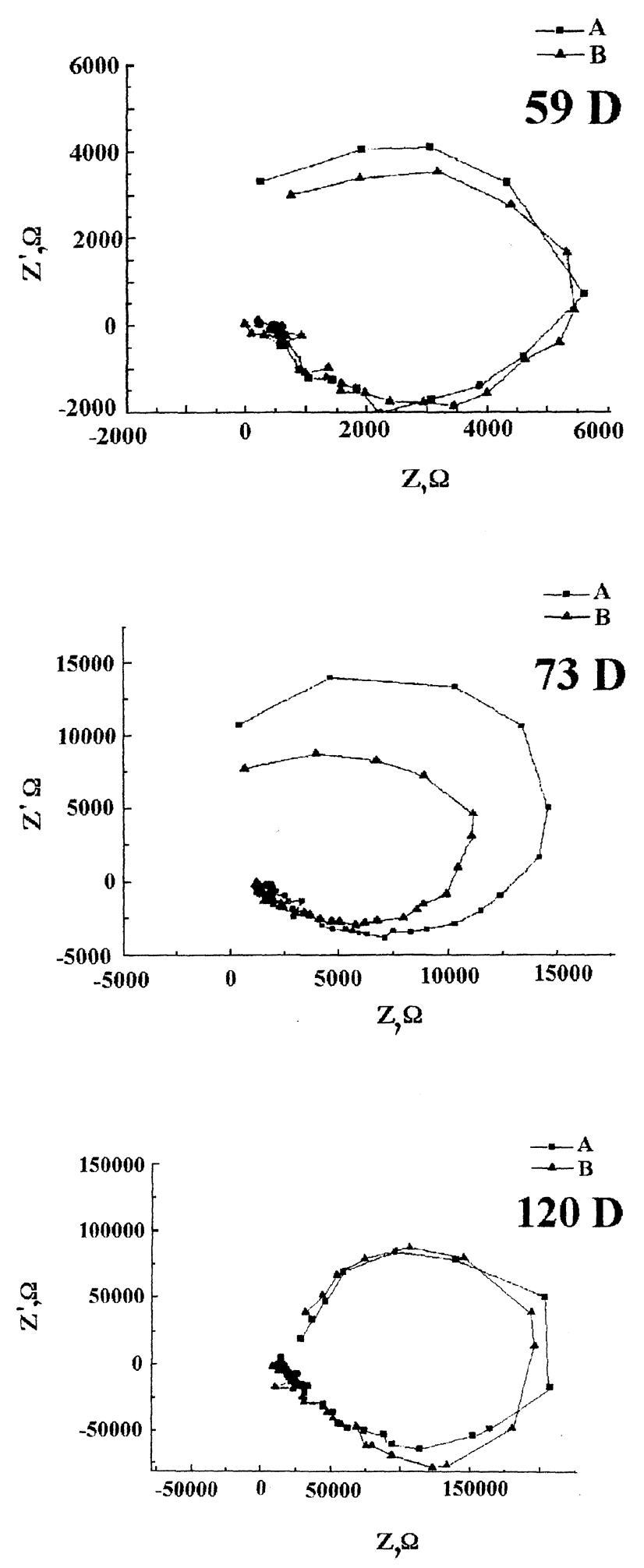

FIG. 3.- Ensayos de impedancia a diferentes tiempos $\left(65 \mathrm{~cm}^{2}\right)$ A: probeta de hormigón polimérico. $\mathrm{B}$ : Probeta de hormigón con recubrimiento de hormigón polimérico.

FIG. 3.- Impedance tests from different times $(65$ $\left.\mathrm{cm}^{2}\right)$ A: specimen of polimeric concrete. $B$ : specimen of concrete with cover of polimeric concrete.

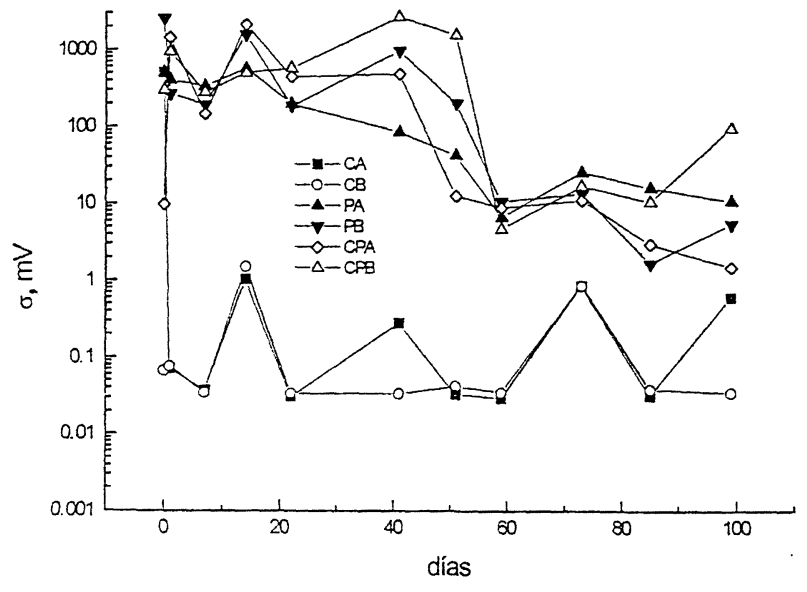

FIG. 4.- Desviación estándar de la señal de ruido en potencial. CA: probeta de hormigón A. CB: probeta de hormigón B. PA: probeta de hormigón polimérico A. PB: probeta de hormigón polimérico B. CPA: probeta de hormigón con recubrimiento de hormigón polimérico A. CPB: probeta de hormigón con recubrimiento de hormigón polimérico $\mathrm{B}$.

FIG. 4.- Standard desviation of the noise sign in potential. $C A$ : specimen of concrete $A$. $C B$ : specimen of concrete $B$. PA: specimen of polimeric concrete A. PB: specimen of polimeric concrete $B$. CPA: specimen of concrete with cover of polimeric concrete A. CPB: specimen of concrete with cover of polimeric concrete $B$.

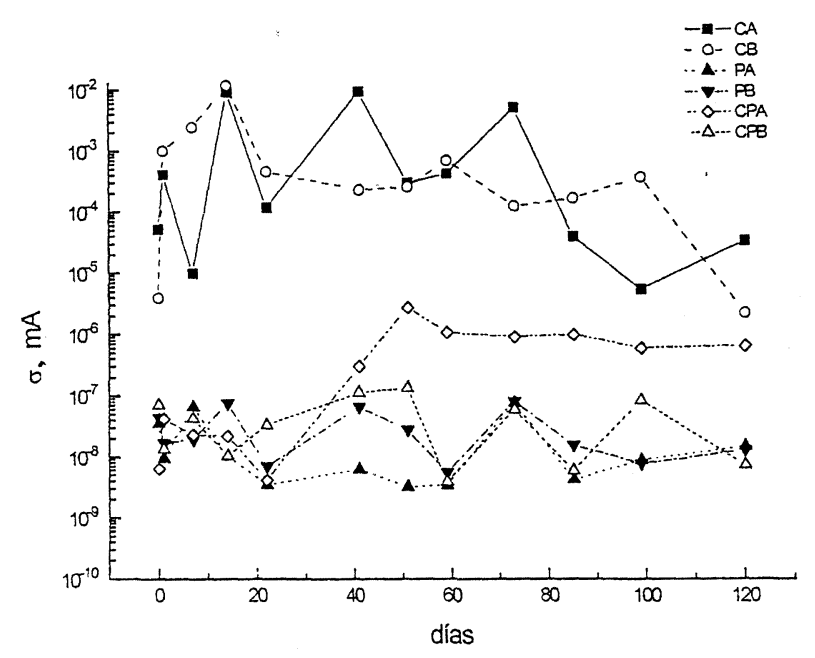

FIG. 5.- Desviación estándar de la señal de ruido en corriente $\left(65 \mathrm{~cm}^{2}\right)$. CA: probeta de hormigón A. CB: probeta de hormigón $\mathrm{B}$. PA: probeta de hormigón polimérico A. PB: probeta de hormigón polimérico B. CPA: probeta de hormigón con recubrimiento de hormigón polimérico A. CPB: probeta de hormigón con recubrimiento de hormigón polimérico $\mathrm{B}$.

FIG. 5.- Standard desviation of the noise sign in current $\left(65 \mathrm{~cm}^{2}\right)$. CA: specimen of concrete $A$. $C B$ : specimen of concrete B. PA: specimen of polimeric concrete A. PB: specimen of polimeric concrete $B$. CPA: specimen of concrete with cover of polimeric concrete A. CPB: specimen of concrete with cover of polimeric concrete $B$. 


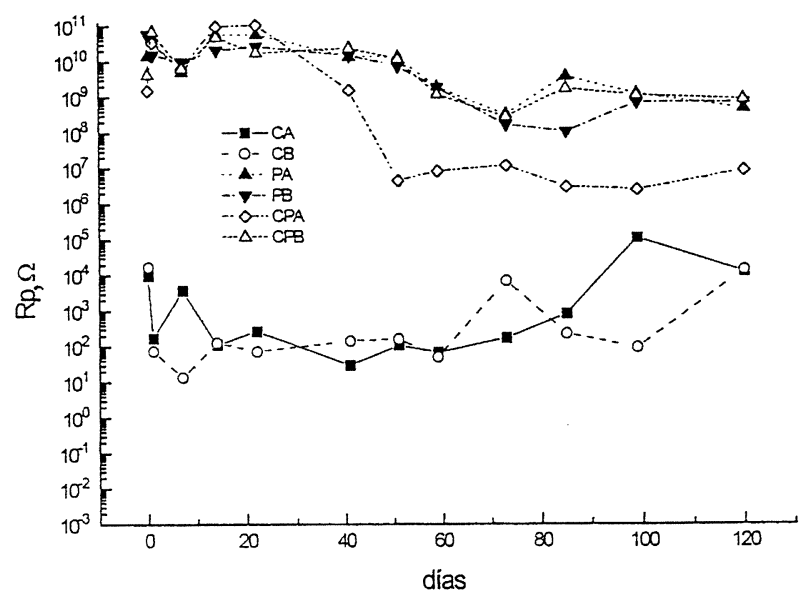

FIG. 6. - Resistencia de polarización $\left(65 \mathrm{~cm}^{2}\right)$. CA: probeta de hormigón $\mathrm{A}$. $\mathrm{CB}$ : probeta de hormigón B. PA: probeta de hormigón polimérico A. PB: probeta de hormigón polimérico B. CPA: probeta de hormigón con recubrimiento de hormigón polimérico A. CPB: probeta de hormigón con recubrimiento de hormigón polimérico $\mathrm{B}$.

FIG. 6.- Polarization resistance $\left(65 \mathrm{~cm}^{2}\right)$. CA: specimen of concrete $A$. $C B$ : specimen of concrete $B$. PA: specimen of polimeric concrete $A$. PB: specimen of polimeric concrete B. CPA: specimen of concrete with cover of polimeric concrete A. $C P B$ : specimen of concrete with cover of polimeric concrete $B$.

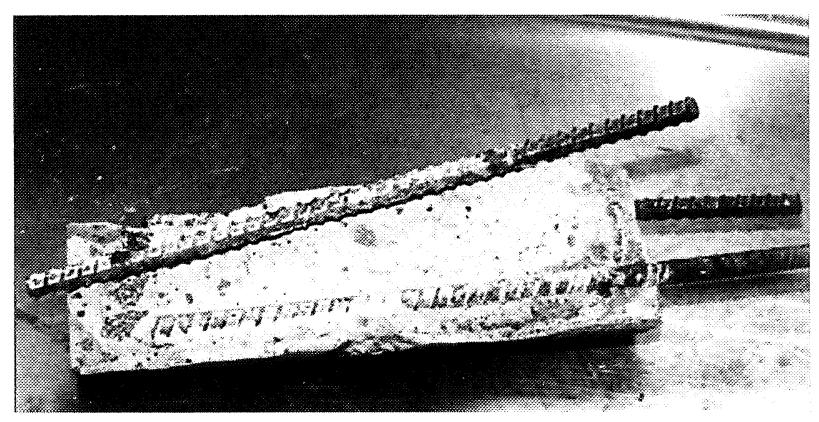

Fig. 7.- Probetas de hormigón normal al final de la prueba.

FIG. 7.- Normal concrete specimens at the end of the test.

- En cambio, el fenómeno de corrosión en hormigón polimérico es totalmente diferente dado que no se pudo determinar la impedancia hasta que permeó el electrólito. En los ensayos de impedancia se aprecia una adsorción enérgica en la superficie de la varilla metálica, indicativa de que el hormigón polimérico ofrece dos tipos de protección contra la corrosión, uno físico, ais-

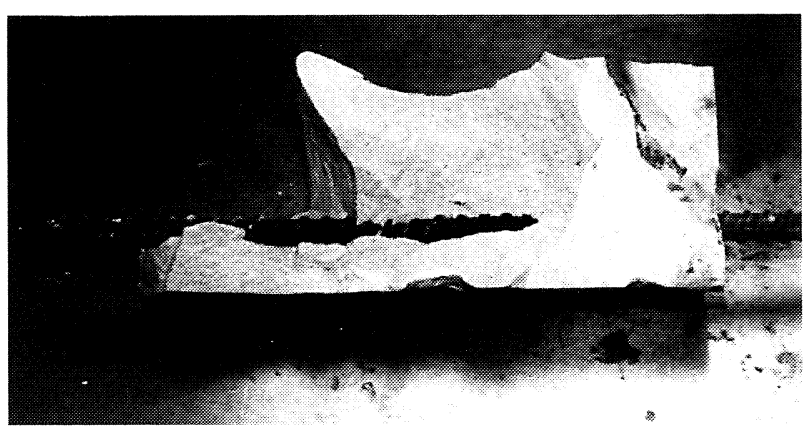

FIG. 8.- Probeta de hormigón polimérico al final de la prueba.

FIG. 8.- Polimeric concrete specimen at the end of the test.

lando en cierta medida el electrólito de la varilla, y otro químico, provocando una inhibición de la corrosión.

- La técnica de ruido electroquímico en potencial y en corriente fue muy adecuada para determinar el tiempo de permeación del electrólito en las probetas con protección de hormigón polimérico, así como la evaluación de dicha protección que es de, al menos, tres órdenes de magnitud. Algo muy significativo fue poder detectar mal recubrimiento en probetas, circunstancia muy importante para detectar fallas.

\section{Agradecimiento}

El autor desea hacer patente su agradecimiento al Dr. Juan Genescá Ll., de la Universidad Nacional Autónoma de México, por sus atinadas observaciones.

\section{REFERENCIAS}

(1) Tracking-resistant electrical insulators containing silica and alumina filler in a polyester resin matrix. Pat. US-5426145.

(2) Andrade C. Rev. Iberoam. Corros. Prot., 15 (4), 1984: $27-$ 34.

(3) Andrade C., Gonzalez, J.A. Rev. Iberoam. Corros. Prot. 15 (4), 1984: 35-41.

(4) Locke, C.E. Solving Corrosion Problems in Concrete. NACE. Preprint No. 2 2/1-2/10. Chicago (EE.UU.), 1982.

(5) Boah, J.K., Somuah, S.K. y LeBlanc, P. Corros. Sci., 46, 1990: 153-159.

(6) Hladky, K. y Dawson, J.L. Corros. Sci., 22, 1982: 231-237.

(7) Chen, J.F. y Bogaerts, W.F. Corros. Sci., 37 (11) 1995: 1.839-1.842.

(8) Eden, D.A., Hladky, K., John, D.G. y Dawson, J.L. Int. Conf. Corrosion '86. Paper 274. NACE. 1986.

(9) Chen, C.T. y Skerry, B.S. Corrosion (Houston), 47 (8), 1991: 598-611. 